\title{
The polarized emission from the galactic plane at arcminute angular resolution
}

\author{
Tom L. Landecker ${ }^{1}$, W. Reich ${ }^{2}$, R. I. Reid ${ }^{1} \dagger$, P. Reich ${ }^{2}$, \\ M. Wolleben ${ }^{1,2}$, R. Kothes ${ }^{1,3}$, D. Del Rizzo ${ }^{1}$, B. Uyanıker ${ }^{1} \ddagger$, \\ A. D. Gray ${ }^{1}$, E. Fürst ${ }^{2}$, A. R. Taylor ${ }^{3}$, and R. Wielebinski ${ }^{2}$
${ }^{1}$ National Research Council of Canada, Herzberg Institute of Astrophysics, Dominion Radio Astrophysical Observatory, P.O. Box 248, Penticton, British Columbia, V2A 6J9, Canada
${ }^{2}$ Max-Planck-Institut für Radioastronomie, Auf dem Hügel 69, 53121 Bonn, Germany
${ }^{3}$ Department of Physics and Astronomy, University of Calgary, 2500 University Drive N.W., Calgary, AB, Canada

\begin{abstract}
As part of the Canadian Galactic Plane Survey (CGPS) we have imaged the polarized emission from the plane of the Milky Way at $1420 \mathrm{MHz}$, covering 1200 square degrees with arcminute resolution. Structure on all scales is represented by combining aperture-synthesis data with single-antenna data. The survey depicts the Magneto-Ionic Medium at a resolution that matches images of other components of the Interstellar Medium within the CGPS database (http://www4.cadc.hia.nrc.gc.ca).
\end{abstract}

Keywords. Polarization - Techniques: polarimetric - Surveys - ISM: magnetic fields

\section{A new survey}

We describe a survey of the polarized emission at $1420 \mathrm{MHz}$, the first extensive survey to combine aperture-synthesis data (from the DRAO Synthesis Telescope - Landecker et al. 2000) with single-antenna data (from the Effelsberg 100-m Telescope - Reich et al. 2004 - and from the DRAO 26-m Telescope - Wolleben et al. 2006). The survey images accurately portray all polarized emission features from the broadest scales to the limit imposed by the angular resolution, $\sim 1^{\prime}$. Data on structures of size $S>40^{\prime}$ comes mainly from the 26-m Telescope, $40^{\prime}>S>15^{\prime}$ from the 100-m Telescope, and $15^{\prime}>S>1^{\prime}$ from the Synthesis Telescope. The survey covers $\ell=65^{\circ}$ to $\ell=175^{\circ}$ over a range $-3.5^{\circ}<b<5.5^{\circ}$ along the northern Galactic plane, with a high-latitude extension from $\ell=105^{\circ}$ to $\ell=120^{\circ}$ up to $b=20^{\circ}$. This survey (Landecker et al. in prep.) is a component of the Canadian Galactic Plane Survey (CGPS - Taylor et al. 2003).

\section{The data}

With $1.7 \times 10^{7}$ independent data points, this is the largest polarization survey made to date, but we can present only a small region here (see Fig. 1). Polarized intensity (PI) is high in the top right corner of Fig. 1, and structure is smooth: this is part of the Fan region seen prominently in single-antenna surveys (e.g. Wolleben et al. 2006). Superposition of other features lowers PI across the rest of the image. We focus our attention on three

$\dagger$ Present address: National Radio Astronomy Observatory, 520 Edgemont Rd., Charlottesville, VA, 22903, USA

$\ddagger$ Present address: 35/3737 Gellatly Road, Westbank, British Columbia, V2T 2W8, Canada 


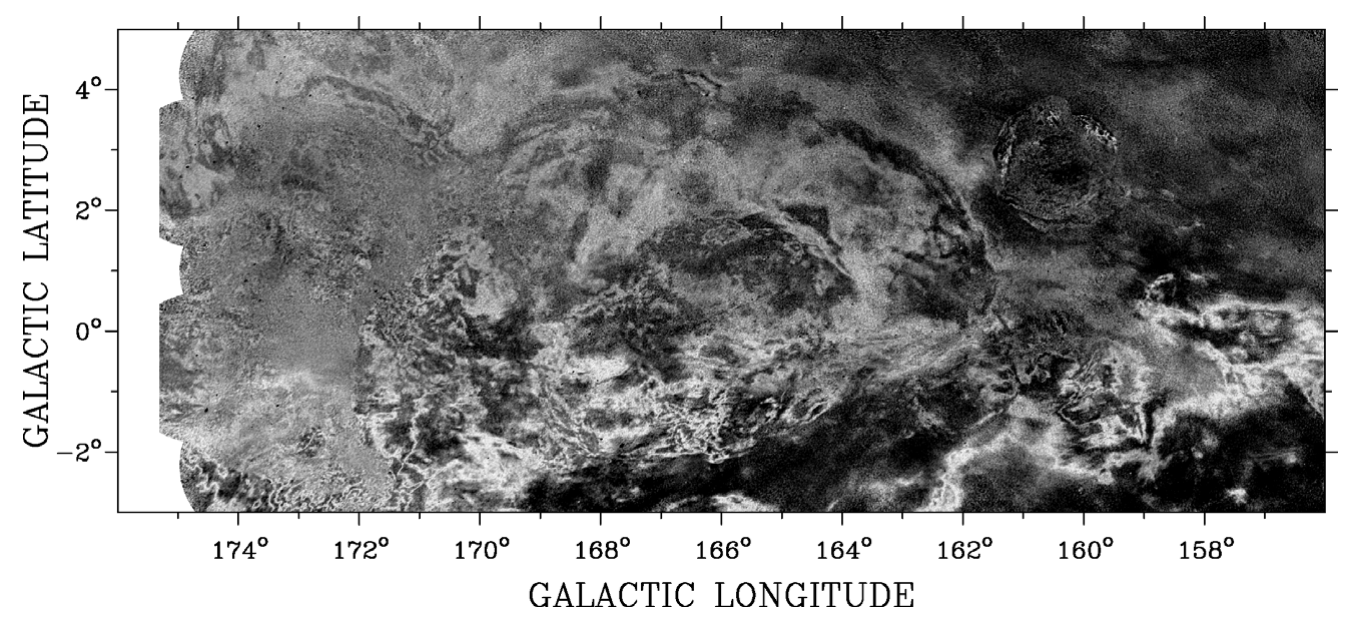

Figure 1. Polarized intensity along the Galactic plane from $\ell=156^{\circ}$ to $\ell=175^{\circ}$. The intensity scale is linear from zero (white) to $500 \mathrm{mK}$ (black).

distinct objects seen in Fig. 1. They are different in nature, and illustrate the diversity of phenomena that can be studied with polarization data of this quality.

The supernova remnant (SNR) HB9 (G160.9+2.6) is seen prominently as a polarized feature, but as a decrease in PI relative to its surroundings. The superposition of polarized SNR emission on a polarized background (or foreground) leads to this reduction.

The planetary nebula Sharpless 2-216 (G158.4+0.2) generates a polarization feature through Faraday rotation in its ionized shell (Ransom et al. 2008). The ionized shell, of density $n_{e} \approx 8 \mathrm{~cm}^{-3}$, generates a barely detectable feature in total intensity, but a strong Faraday rotation signature, with $\Delta \psi \approx 110^{\circ}$, produced in a field whose line-of-sight component is $\sim 5 \mu \mathrm{G}$. This bears witness to the sensitivity of polarization measurements.

The third object would not have been discovered by any means other than its Faraday rotation signature. It is seen in Fig. 1 as a large shell, at least $8^{\circ}$ in extent, centered at $\ell=166^{\circ}, b=-1^{\circ}$. Association with an atomic hydrogen shell at $v_{l s r}=-20 \mathrm{~km} \mathrm{~s}^{-1}$ places the object in the Perseus Arm at a distance of $\sim 2 \mathrm{kpc}$. Kothes et al. (in preparation) interpret this object as a stellar-wind bubble whose extent is some $350 \mathrm{pc}$. Once identified through its polarization signature, the object can be recognized in other wavebands.

We conclude from this remarkable result that (a) Faraday rotation is a powerful tool for the detection of ionized gas, (b) there are Galactic objects many degrees in extent which can only be detected in data with arcminute resolution, and (c) the data for this object would be impossible to interpret without the incorporation of single-antenna data into the polarization images. Further work on the survey will include correlations of polarization features with other ISM tracers, studies of depolarization by foreground HII regions, and statistical (power-spectrum and structure-function) studies.

\section{References}

Landecker, T. L., Dewdney, P., Burgess, T. A., et al. 2000, A\&AS 145, 509

Ransom, R., Uyanıker, B., Kothes, R., \& Landecker, T.L. 2008, ApJ 684, 1009

Reich, W., Fürst, E., Reich, P., et al. 2004, in The Magnetized Interstellar Medium, ed.

B. Uyanıker, W. Reich, \& R. Wielebinski, 45

Taylor, A. R., Gibson, S. J., Peracaula, M., et al. 2003, AJ 125, 3145

Wolleben, M., Landecker, T. L., Reich, W., \& Wielebinski, R. 2006, A\&\&A 448, 411 